\title{
Dexmedetomidine and remifentanil in the perioperative management of an adolescent undergoing resection of pheochromocytoma
}

\section{-A case report-}

\author{
Jae-Wook Jung ${ }^{1}$, Jung Kyu Park ${ }^{2}$, Sang Yoon Jeon ${ }^{1}$, Yong Han Kim ${ }^{1}$, So-Hyun Nam³ , Young-Gyun Choi ${ }^{1}$, \\ and Si Ra Bang ${ }^{1}$ \\ Department of Anesthesiology and Pain Medicine, ${ }^{1}$ Haeundae Paik Hospital, College of Medicine, Inje University, ${ }^{2}$ Busan Paik \\ Hospital, College of Medicine, Inje University, ${ }^{3}$ Department of Surgery, Haeundae Paik Hospital, College of Medicine, Inje University, \\ Busan, Korea
}

A 15-year-old adolescent with unilateral multiple adrenal pheochromocytoma had an episode of subcortical intracerebral hemorrhage and seizure 6 weeks before the surgery. He was pretreated with terazosin, losartan, atenolol and levetiracetam for 2 weeks. Dexmedetomidine was started in the preoperative waiting area, and a combination of dexmedetomidine and remifentanil was continuously infused for most of anesthetic time. To control blood pressure, bolus injection of remifentanil and low-dose infusion of sodium nitroprusside, nicardipine, and esmolol were administered during three adrenergic crises. There was minimal post-resection hypotension, and his trachea was extubated safely $20 \mathrm{~min}$ after the surgery. He was discharged without noticeable complication. His catecholamine levels showed the steadily decreasing pattern during the operation in this case. Though a combination of dexmedetomidine and remifentanil may not prevent the hemodynamic instability impeccably during the tumor manipulation, this combination seems to be the way of interrupting release of catecholamines and minimizing hemodynamic fluctuations. (Korean J Anesthesiol 2012; 63: 555-558)

Key Words: Catecholamine, Dexmedetomidine, Pheochromocytoma, Remifentanil.

Received: November 24, 2011. Revised: 1st, December 29, 2011; 2nd, February 7, 2012. Accepted: February 10, 2012.

Corresponding author: Si Ra Bang, M.D., Department of Anesthesiology and Pain Medicine, Haeundae Paik Hospital, College of Medicine, Inje University, 1435, Jwa-dong, Haeundae-gu, Busan 612-030, Korea. Tel: 82-51-797-0421, Fax: 82-51-797-0499, E-mail: sira1045@naver.com (c) This is an open-access article distributed under the terms of the Creative Commons Attribution Non-Commercial License (http:// creativecommons.org/licenses/by-nc/3.0/), which permits unrestricted non-commercial use, distribution, and reproduction in any medium, provided the original work is properly cited. 
Pheochromocytoma is a rare tumor commonly originated from adrenal medulla. If functionally active, it can result in the release of too much epinephrine, norepinephrine, and dopamine that control blood pressure (BP), heart rate (HR), and metabolism [1]. Therefore, the patients could have headache, sweating, palpitations, weight loss, and hypertension. It is only curable when proper surgical resection of tumors is performed. When the operation is perfomed, it is an extreme challenging period to an anesthesiologist, because of its sharp increasing of catecholamine levels and cardiovascular responses. There were many attempts to suppress the release of catecholamines and to attenuate systemic responses during the operation. Dexmedetomidine can reduce the systemic reaction to norepinephrine by activating sympathetic alpha-2 receptor [2]. Remifentanil can also be beneficial in hemodynamic stability [3]. We had an anesthetic management using the combination of dexmedetomidine and remifentanil during resection of unilateral multiple pheochromocytoma in a 15-year-old adolescent who had seizure and intracerebral hemorrhage (ICH) before the surgery.

\section{Case Report}

A 15-year-old adolescent (weight, $46 \mathrm{~kg}$; height, $162 \mathrm{~cm}$ ) had a medical history of hypertension, cyclic headache, and vomiting for 10 years without an awareness of his medical condition. Thereafter, he demonstrated a seizure with subcortical ICH in the left frontal area 6 weeks before the surgery. He was diagnosed with pheochromocytoma as confirmed by excessive catecholamine production in his serum and urine. There was no suspicious lesion to elucidate his seizure with ICH. The computed tomography scan revealed two masses (dimension, $4.0 \times 3.5 \mathrm{~cm}$, and $1.6 \times 1.2 \mathrm{~cm}$ ) in the left adrenal gland .

Preoperative preparation consisted of terazosin $1 \mathrm{mg}$, losartan $1.25 \mathrm{mg}$, atenolol $25 \mathrm{mg}$, and levetiracetam $1 \mathrm{~g}$ daily for 2 weeks, which resulted in symptomatic improvement, normotension and prevention of seizure. Before entering the operating room, 2 $\mathrm{mg}$ of midazolam was infused and dexmedetomidine was given $1 \mu \mathrm{g} / \mathrm{kg}$ over $10 \mathrm{~min}$ at the waiting area, followed by a continuous intravenous infusion at $0.5 \mu \mathrm{g} / \mathrm{kg} / \mathrm{hr}$. On arrival to the operating room, the patient was mildly sedated.

The intraoperative monitoring consisted of electrocardiogram (lead II), HR, pulse oximeter, end-tidal $\mathrm{CO}_{2}$, bispectral index, esophageal temperature, invasive measurement of BP with cardiac index, central venous pressure, central venous oxygen saturation, and urine output. Arterial blood gases, electrolytes, glucose, and serum catecholamines were planned to check before induction and after intubation, tumor manipulation, and removal of tumors. General anesthesia was induced with propofol $100 \mathrm{mg}$, continuous infusion of dexmedetomidine, remifentanil $0.2 \mu \mathrm{g} / \mathrm{kg} / \mathrm{min}$ and sevoflurane 2.0 vol\% by mask. Rocuronium $40 \mathrm{mg}$ was given to facilitate tracheal intubation. The trachea was intubated with no significant hemodynamic changes. Anesthesia was maintained with a $2.5 \%$ end-tidal concentration of sevoflurane in an oxygen-air mixture $\left(\mathrm{FiO}_{2}=0.50\right)$ and infusion of remifentanil $0.1 \mu \mathrm{g} / \mathrm{kg} / \mathrm{min}$ and dexmedetomidine $0.5 \mu \mathrm{g} / \mathrm{kg} /$ $\mathrm{hr}$. Bispectral index was maintained between 32 and 54 during the procedure. There were no remarkable hemodynamic changes before manipulation of a smaller tumor. However, when the surgeon began to manipulate it, BP and HR were abruptly increased (Fig. 1). Those changes were controlled with bolus injection of remifentanil, nicardipine, esmolol, and sodium nitroprusside. There were two more adrenergic surges before ligation of the left adrenal vein. Remifentanil, nicardipine, and sodium nitroprusside were also used to put back his vitals to baseline. After tumor removal, BP decreased rapidly, which responded to ephedrine and phenylephrine to produce an increase in systolic BP of $80-100 \mathrm{mmHg}$. Dexmedetomidine and remifentanil were stopped at closure of peritoneum and at end of skin closure, respectively. The trachea was extubated $20 \mathrm{~min}$ after the end of surgery. After extubation, the patient was mildly sedated but had good respiration and possible purposeful movement. The child received intravenous patientcontrolled analgesia for postoperative pain control. The total operation time was $200 \mathrm{~min}$, and the total period of anesthesia was $285 \mathrm{~min}$. Estimated blood loss was about $200 \mathrm{ml}$ and the total dose of infused dexmedetomidine was $144 \mu \mathrm{g}$. After the surgery, he stayed at the intensive care unit for 1 day and was discharged 10 days after the surgery with no noticeable complications and events.

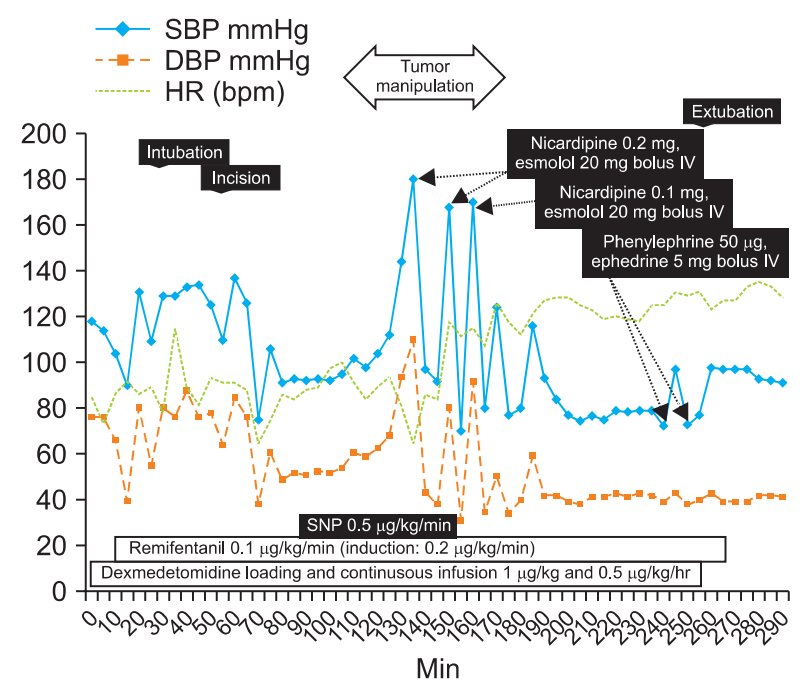

Fig. 1. Perioperative changes in systolic blood pressure (SBP), diastolic blood pressure (DBP) and heart rate (HR). 


\section{Discussion}

The anesthetic management for maintaining stable cardiovascular response during the resection of pheochromocytoma is still an unsolved assignment to the anesthetist. During the operation, catecholamine level increase of up to 200,000$1,000,000 \mathrm{pg} / \mathrm{ml}$ (normal catecholamine levels under stressful events: $200-2,000 \mathrm{pg} / \mathrm{ml}$ ) [4], should be anticipated and avoided. Because our patient had an episode of seizure and ICH only 6 weeks before the surgery, we had to concentrate on keeping his BP under our control to avoid neurologic complications.

Dexmedetomidine is a highly selective alpha-2 adrenoceptor agonist compared with clonidine, especially for the $2_{\mathrm{A}}$ subtype [5]. The alpha-2 adrenoceptors are involved in regulating the autonomic and cardiovascular systems. They are located on blood vessels, where they mediate vasoconstriction, and on sympathetic terminals, where they inhibit noradrenaline release. Alpha-2 adrenoceptors are also located within the central nervous system, and their activation leads to sedation, a $60-80 \%$ reduction of tonic levels of sympathetic outflow and catecholamines, and an augmentation of cardiac-vagal activity [6]. Dexmedetomidine attenuates sympathoadrenal responses to tracheal intubation and surgical stimuli and has a significant anesthetic and analgesic sparing effect when used intraoperatively [7]. Additionally, dexmedetomidine has recently been investigated for its potential in many other clinical scenarios, including neuroprotection, cardioprotection and renoprotection, with promising results $[8,9]$. These advantages make dexmedetomidine an effective adjunct to general anesthesia in patients with pheochromocytoma.

The pharmacokinetics of remifentanil is characterized by small volume of distribution, rapid clearance, and short contextsensitive half-time. Prompt onset and short duration of action make remifentanil a useful selection for the suppression of the transient sympathetic nervous system response to tracheal intubation and noxious stimuli in at risk patients [3]. Although remifentanil is effective for blunting the sympathetic response, there were reports that even if remifentanil was being infused in pheochromocytoma excision to control intraoperative hemodynamic instability, the use of vasodilators was required during tumor manipulations [10]. Although our anesthetic team assumed that the combination of dexmedetomidine and

Table 1. Perioperative Catecholamine Levels

\begin{tabular}{lccc}
\hline & Epinephrine & Norepinephrine & Dopamine \\
\hline Normal range & $<300 \mathrm{pg} / \mathrm{ml}$ & $<800 \mathrm{pg} / \mathrm{ml}$ & $<200 \mathrm{pg} / \mathrm{ml}$ \\
Preoperation & 397 & 8,580 & 69 \\
After intubation & 245 & 4,903 & 101 \\
After vessel ligation & 372 & 2,004 & 71 \\
After adrenalectomy & 214 & 1,215 & 65 \\
\hline
\end{tabular}

remifentanil might produce additional hypotensive agents to be unnecessary, we actually infused low dose of esmolol, sodium nitroprusside and nicardipine when BP surges came up during tumor manipulation. Because one of masses was placed difficult to dissect, the surgeon had a hard time manipulating the tumors before ligation of the adrenal vein.

There are some reports on serum plasma catecholamine levels during the resection of pheochromocytoma $[11,12]$, which show wide variation depending on what stimuli had been done to the patient. In comparison, our patient's levels of catecholamine (Table 1) represented persistent decreasing trend except with epinephrine, which means that infusion of dexmedetomidine and remifentanil might interrupt the release of norepinephrine effectively during the procedure. Russell et al. [13], in a retrospective analysis of a group of adult patients, found a significant correlation between intraoperative cardiovascular instability and peak serum catecholamine levels measured at the time. Mikasa et al. [14] also showed a significant correlation between plasma norepinephrine concentration and mean arterial pressure during surgery. Bryskin and Weldon [15] reported that the infusion of dexmedetomidine and magnesium sulfate in their case was effective for stable hemodynamics, with the pattern of steadily decreasing catecholamine levels following intubation. Therefore, we presume that the decreasing trend of catecholamine levels could help to diminish sudden fluctuating changes of hemodynamics.

In conclusion, the levels of perioperative catecholamines demonstrate that the combination of dexmedetomidine and remifentanil was an effective manner to achieve safe anesthesia in our patient. The optimal intra-operative dosing and timing of dexmedetomidine and remifentanil remain to be determined, and a further study in this setting is needed.

\section{References}

1. Prys-Roberts C. Phaeochromocytoma-recent progress in its management. Br J Anaesth 2000; 85: 44-57.

2. Bravo EL. Pheochromocytoma: an approach to antihypertensive management. Ann N Y Acad Sci 2002; 970: 1-10.

3. Thompson JP, Hall AP, Russell J, Cagney B, Rowbotham DJ. Effect of remifentanil on the haemodynamic response to orotracheal intubation. Br J Anaesth 1998; 80: 467-9.

4. Roizen MF, Fleisher LA. Anesthetic Implications of Concurrent Diseases. In: Anesthesia. 7th ed. Edited by Miller RD: Philadelphia, Churchill Livingstone. 2010, pp 1084-5.

5. Chrysostomou C, Schmitt CG. Dexmedetomidine: sedation, analgesia and beyond. Expert Opin Drug Metab Toxicol 2008; 4: 619-27.

6. Kallio A, Scheinin M, Koulu M, Ponkilainen R, Ruskoaho H, Viinamäki $\mathrm{O}$, et al. Effects of dexmedetomidine, a selective alpha 2-adrenoceptor agonist, on hemodynamic control mechanisms. Clin Pharmacol Ther 1989; 46: 33-42.

7. Scheinin B, Lindgren L, Randell T, Scheinin H, Scheinin M. 
Dexmedetomidine attenuates sympathoadrenal responses to tracheal intubation and reduces the need for thiopentone and perioperative fentanyl. Br J Anaesth 1992; 68: 126-31.

8. Ruesch S, Levy JH. Treatment of persistent tachycardia with dexmedetomidine during off-pump cardiac surgery. Anesth Analg 2002; 95: 316-8.

9. Ma D, Rajakumaraswamy N, Maze M. alpha2-Adrenoceptor agonists: shedding light on neuroprotection? Br Med Bull 2005; 71: 77-92.

10. Baraka A, Siddik S, Alameddine M. Remifentanil for modulation of hemodynamics in a patient undergoing laparoscopic resection of pheochromocytoma. Middle East J Anesthesiol 2004; 17: 585-92.

11. Tauzin-Fin P, Sesay M, Gosse P, Ballanger P. Effects of perioperative alphal block on haemodynamic control during laparoscopic surgery for phaeochromocytoma. Br J Anaesth 2004; 92: 512-7.
12. Pretorius M, Rasmussen GE, Holcomb GW. Hemodynamic and catecholamine responses to a laparoscopic adrenalectomy for pheochromocytoma in a pediatric patient. Anesth Analg 1998; 87: 1268-70.

13. Russell WJ, Metcalfe IR, Tonkin AL, Frewin DB. The preoperative management of phaeochromocytoma. Anaesth Intensive Care 1998; 26: 196-200.

14. Mikasa H, Sakuragi T, Higa K, Yasumoto M. Skin blood flow and plasma catecholamine concentrating during removal of a phaeochromocytoma in a child. Br J Anaesth 2004; 92: 757-60.

15. Bryskin R, Weldon BC. Dexmedetomidine and magnesium sulfate in the perioperative management of a child undergoing laparoscopic resection of bilateral pheochromocytomas. J Clin Anesth 2010; 22: 126-9. 